\title{
Ipriflavone attenuates the degeneration of cartilage by blocking the Indian hedgehog pathway
}

Li Guo', Xiaochun Wei ${ }^{1}$, Zhiwei Zhang ${ }^{1}$, Xiaojian Wang ${ }^{1}$, Chunli Wang ${ }^{1}$, Pengcui Li ${ }^{1}$, Chunfang Wang ${ }^{2}$ and Lei $\mathrm{Wei}^{1,3^{*}}$

\begin{abstract}
Background: To determine if ipriflavone, a novel and safe inhibitor of Indian hedgehog (Ihh) signaling, can attenuate cartilage degeneration by blocking the Ihh pathway.

Methods: Human chondrocytes were used to evaluate Ihh signaling, cell proliferation, apoptosis, gene, and protein expression of chondrocytes by cell proliferation and apoptosis assays, real-time $\mathrm{qPCR}$, and Western blotting at $48 \mathrm{~h}$ after ipriflavone treatment. Human cartilage explants were further used to validate the cell culture results. The effects of ipriflavone on cartilage degeneration in vivo were assessed using the rat ACLT OA model. Two-month-old male SD rats were randomized into 3 groups $(n=75)$ : (1) sham, (2) ACLT alone, and (3) ACLT+ ipriflavone. Ipriflavone was administered intragastrically at $24 \mathrm{~h}$ after ACLT for 6 weeks. The extent of OA progression was evaluated by the OARSI score and immunohistochemistry at 12 weeks after surgery. The Ihh signaling pathway and OA-related genes were quantified by real-time PCR.

Results: Cell proliferation in the cells treated with ipriflavone was increased to $36.40 \% \pm 1.32 \%(5 \mu \mathrm{M})$ and $28.54 \% \pm$ $0.74 \%(10 \mu M)$ from $11.99 \% \pm 0.35 \%$ (DMSO) $(P<0.001)$, and apoptosis was decreased to $12.64 \% \pm 3.7 \%(5 \mu M)$ and $15.18 \% \pm 3.13 \%(10 \mu \mathrm{M})$ from $25.76 \% \pm 5.1 \%$ (DMSO) $(P<0.05)$. Ipriflavone blocked Runx-2 mainly through the SmoGli2 pathway. A similar result was found in the cartilage explant culture. Ihh signaling in vivo was inhibited in animals treated with ipriflavone. Safranin-O staining revealed a less cartilage damage with lower OARSI scores $(P<$ $0.05)$ in the ipriflavone-treated animals compared with untreated animals. The gene expression of Smo and Gli2 was inhibited significantly by ipriflavone $(P<0.05)$. The OA-related gene and protein type $X, M M P-13$, and type II collagen-C fragment were reduced, while type II collagen and Agg were increased in the ipriflavone-treated animals $(P<0.05)$.
\end{abstract}

Conclusions: Catabolic genes were disrupted by blocking the Ihh pathway. This finding suggests that disruption of Ihh signaling with ipriflavone provides chondral protection in rat posttraumatic OA.

Keywords: Ipriflavone, Osteoarthritis, Indian hedgehog, Chondrocyte hypertrophy

\footnotetext{
* Correspondence: lei_wei@brown.edu

${ }^{1}$ Department of Orthopedics, Second Hospital of Shanxi Medical University,

Shanxi Key Laboratory of Bone and Soft Tissue Injury Repair, Taiyuan, China

No. 382, Wuyi Road, Taiyuan 030001, China

${ }^{3}$ Department of Orthopedics, Warren Alpert Medical School of Brown

University, Suite 402A, 1 Hoppin Street, Providence, RI 02903, USA

Full list of author information is available at the end of the article
}

(c) The Author(s). 2019 Open Access This article is distributed under the terms of the Creative Commons Attribution 4.0 International License (http://creativecommons.org/licenses/by/4.0/), which permits unrestricted use, distribution, and reproduction in any medium, provided you give appropriate credit to the original author(s) and the source, provide a link to the Creative Commons license, and indicate if changes were made. The Creative Commons Public Domain Dedication waiver (http://creativecommons.org/publicdomain/zero/1.0/) applies to the data made available in this article, unless otherwise stated. 


\section{Background}

Osteoarthritis (OA) is one of the most common musculoskeletal diseases with aging. OA is characterized by progressive degeneration of the articular cartilage and joint swelling, pain, stiffness, and loss of mobility. These characteristics not only significantly affect the quality of life but also aggravate the financial burden of patients [1]. However, the underlying molecular mechanisms involved in the pathogenesis and progression of OA are still unknown, and no disease-modifying therapy is available.

Recently, we and others found a significant increase in Indian hedgehog (Ihh) protein expression in human osteoarthritic cartilage, which promotes chondrocyte hypertrophy and the upregulation of matrix metallopeptidase 13 (MMP-13) [2-7]. We further demonstrated that disrupting the Ihh signaling pathway in vivo attenuates surgically induced OA progression in Col2a1-CreERT2; Ihh fl/fl mice [8]. This evidence indicates that upregulation of the Ihh pathway plays a key role during $\mathrm{OA}$ progression and that inhibiting the Ihh pathway may attenuate the degeneration of cartilage. However, Ihh gene deletion is not a therapeutic option in humans, and most chemical inhibitors of hedgehog (Hh) signaling induce severe side effects, including holoprosencephaly, cleft palate, and limb defects [9-13]. Therefore, a safe inhibitor of the Hh pathway would be extremely important for human OA treatment.

Recently, three Hh pathway inhibitors have been identified among 4240 compounds using small molecule screening: (1) ipriflavone, a dietary supplement; (2) tolnaftate, an antifungal agent; (3) and 17-b-estradiol, a human hormone and pharmaceutical agent [14]. These compounds demonstrate similar efficiencies of Hh signaling inhibition in both mouse and human cells without cytotoxicity, and they are 8- to 30 -fold safer than the index Hh pathway inhibitor cyclopamine. For this study, we choose ipriflavone, a synthetic isoflavone that is used worldwide as a supplement for its touted anabolic and bone density-building properties [15-19] to test our hypothesis: blockade of the Ihh pathway with ipriflavone alleviates the degeneration of cartilage in vitro and in vivo because it has been used to treat diseases, such as breast cancer and diabetes in rats via inhibiting the Ihh pathway [20,21], and the pharmacokinetics of Ipriflavone have been described in animal models [22] and humans [23].

\section{Methods}

\section{Chondrocyte isolation and primary culture}

This study was approved by the Institutional Animal Care and Use Committee of the Second Hospital of Shanxi Medical University (CMTT\#: 2013025). Cartilage slices were removed from the "relatively normal" cartilage samples of the tibia obtained during total knee arthroplasty and washed in Dulbecco's modified Eagle's medium
(DMEM) (Invitrogen, Carlsbad, CA, USA). Chondrocytes were isolated as previously described [24]. Briefly, pieces of cartilage were minced with a scalpel and digested with pronase $(2 \mathrm{mg} / \mathrm{ml})$ (Roche, Basel, Switzerland) in Hank's Balanced Salt Solution (HBSS) (Invitrogen, Carlsbad, CA, USA) for $30 \mathrm{~min}$ at $37^{\circ} \mathrm{C}$ with shaking. After digestion and removal of the supernatant, the cartilage pieces were washed with DMEM and digested with crude bacterial collagenase (type IA, $1 \mathrm{mg} / \mathrm{ml}$ ) (Sigma-Aldrich, St Louis, $\mathrm{MO}$, USA) for $6-8 \mathrm{~h}$ at $37^{\circ} \mathrm{C}$ with shaking. Enzymatic digestion was stopped by adding DMEM containing $10 \%$ fetal bovine serum (FBS) (Invitrogen, Carlsbad, CA, USA). Residual multicellular aggregates were removed by filtering, and the cells were washed three times with DMEM. Primary chondrocytes were incubated in DMEM containing $10 \%$ FBS, L-glutamine (Invitrogen, Carlsbad, CA, USA), and antibiotics (penicillin and streptomycin) (Sigma-Aldrich, St Louis, MO, USA) under $37^{\circ} \mathrm{C}, 5 \% \mathrm{CO}_{2}$ condition and allowed to attach to the surface of the culture dishes (Nalge Nunc International Corp, Naperville, IL, USA). At 90\% confluence, the cells were treated with ipriflavone (Sigma-Aldrich, St Louis, MO, USA) dissolved in dimethyl sulfoxide (DMSO) (Sigma-Aldrich, St Louis, $\mathrm{MO}, \mathrm{USA})$ to a concentration of $5 \mu \mathrm{M}$ and $10 \mu \mathrm{M}$, and the $0.1 \%$ DMSO treatment group was used as the control. After $48 \mathrm{~h}$ in culture without removing the reagent, the cell proliferation assay, cell apoptosis assay, total RN, and total protein were isolated from the chondrocytes. Immunocytochemistry analyses of chondrocyte phenotypes were performed as described previously using anti-type I collagen (SC-59772, Santa Cruz, CA, USA) and anti-type II collagen monoclonal antibodies (SC-52658, Santa Cruz, CA, USA) [25]. These cells were positive for type II collagen and negative for type I collagen staining (Fig. 1A). In addition, cell proliferation and apoptosis assays were also conducted using chondrocytes.

\section{Human cartilage explant culture}

Cartilage samples were obtained from the "relatively normal" cartilage samples of the tibial plateau obtained during total knee arthroplasty. The cartilage samples were then cut into $4 \mathrm{~mm}^{3}$ pieces weighing 6-9 $\mathrm{mg}$ each with a scalpel and cultured in DMEM/F12 supplemented with $10 \%$ FBS. Among the collected explants, we randomly subdivided them into three groups: treatment with $50 \mu \mathrm{M}$ ipriflavone, $100 \mu \mathrm{M}$ ipriflavone, and $0.1 \% \mathrm{DMSO}$ as the control (Fig. 3A). After $72 \mathrm{~h}$ of treatment without removing the reagent, the total mRNA and total protein were isolated from the cartilage tissues, respectively.

\section{Rat anterior cruciate ligament transection (ACLT) model of $O A$ treated with ipriflavone}

Two-month-old male SD rats (180-230 g, $n=75)$ were obtained from the Experimental Animal Centre, Shanxi 

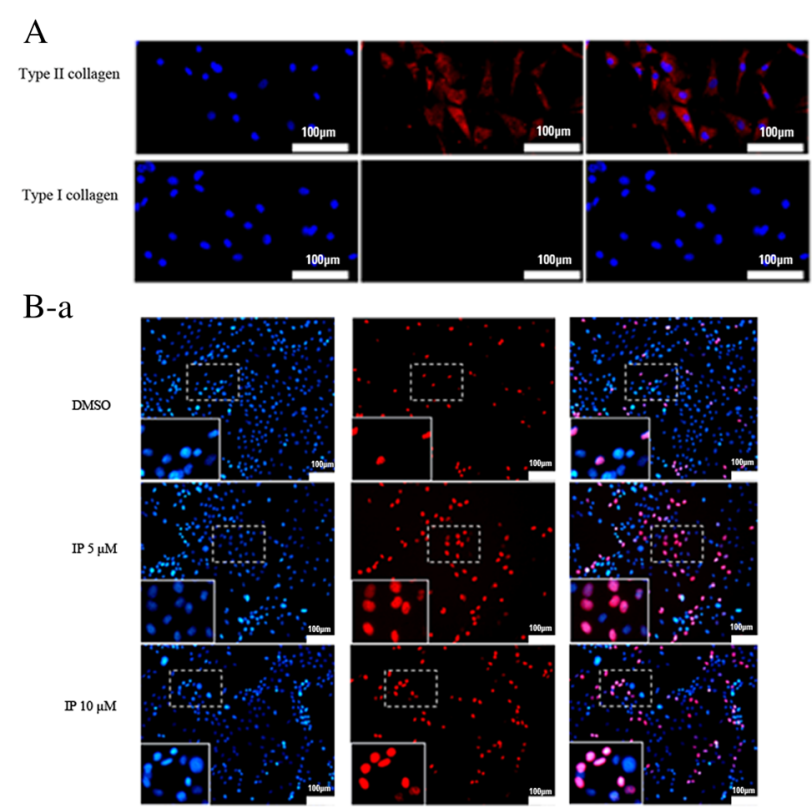

$3-b$

C-a
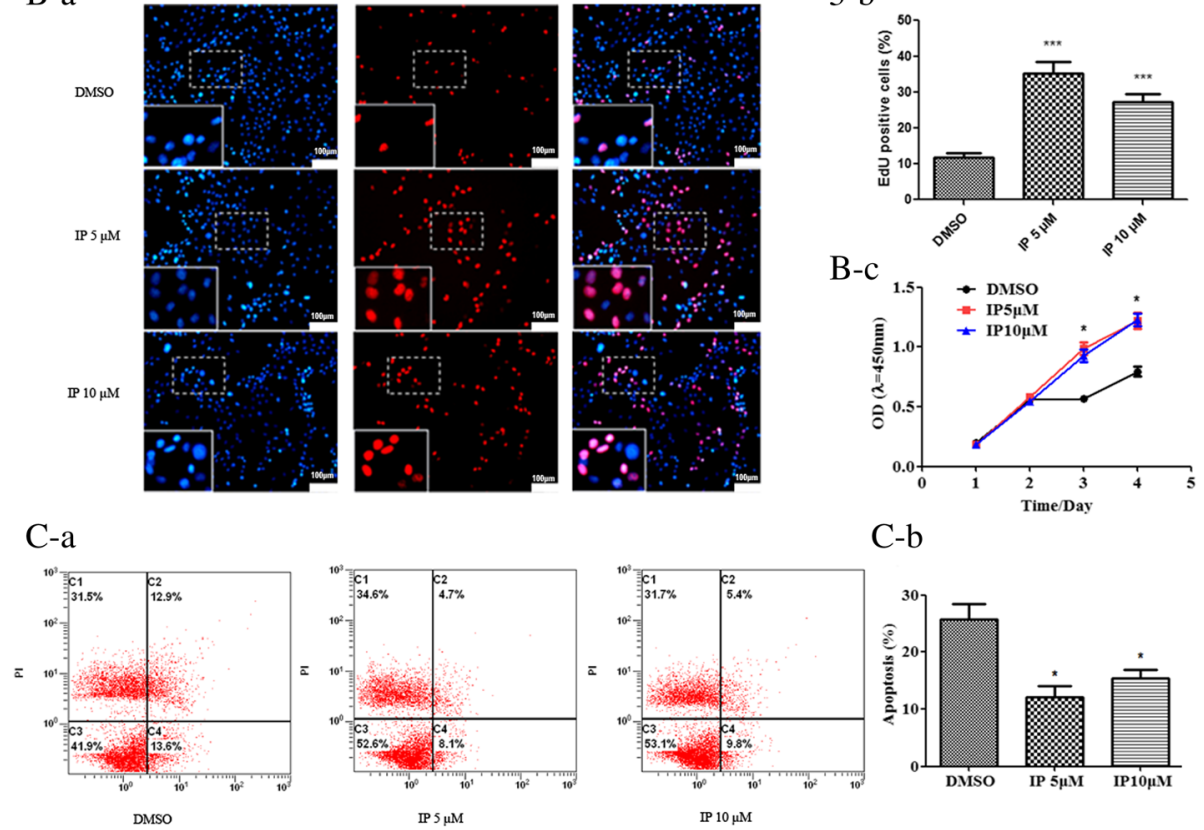

C-b

Fig. 1 Ipriflavone promoted the proliferation and reduced the apoptosis of human chondrocytes. A, The results of the immunofluorescence assay showed that the cells (primary human chondrocytes were incubated in DMEM supplemented with 10\% FBS in the presence or absence of ipriflavone) were positive for type II collagen and negative for type I collagen staining (red, scale bar: $100 \mu \mathrm{m}$ ), and the cultured cells maintained their chondrocytes phenotype. B-a, The EdU-based cell proliferation assay showed that compared with the DMSO group, and the EdU-positive cells (red, scale bar: $100 \mu \mathrm{m}$ ) were significantly increased in both the $5 \mu \mathrm{M}$ and $10 \mu \mathrm{M} I \mathrm{P}$ treatment groups. B-b, The percentage of EdU-positive cells was quantified, and the proliferation of chondrocytes was significantly increased in both IP treatment groups. Data are expressed as means \pm SDs $(n=6){ }^{* * *} P<0.001$ versus the DMSO group. B-c, The CCK-8 assay results showed that the viability of chondrocytes was higher in both IP treatment groups than the DMSO control group, and the viability gradually increased with a longer treatment time. Thus, IP significantly promotes the proliferation of human chondrocytes in vivo. C, The Annexin V-FITC/propidium iodide (PI) dual staining assay by flow cytometry indicated that apoptosis was reduced in the IP treatment group compared with the DMSO control group at $48 \mathrm{~h}$ after treatment. Values are the mean \pm SDs. $(n=3) * P<0.05$ versus the DMSO group

Medical University, China. The rats were housed under specific pathogen-free conditions with a 12-h/12-h light/ dark cycle. All animals were handled and used in accordance with the Guidelines for the Use and Care of Laboratory Animals provided by Shanxi Medical University. All animal experiments were approved by the Institutional Review Board at the Second Hospital of Shanxi Medical University (Taiyuan, China; CMTT\#: 2013012, Approval 2013).

The rats were randomized to 3 groups $(n=25$ per group): (1) sham operation, (2) ACLT alone, and (3) ACLT and $200 \mathrm{mg} / \mathrm{kg}$ ipriflavone [21, 22]. ACLT and sham operations were performed on the right knees as described previously [26]. Ipriflavone was used to treat rat posttraumatic osteoarthritis (PTOA) by intragastrical administration. The treatment was performed $24 \mathrm{~h}$ after ACLT and then once a day for 6 weeks. All animals were euthanized 12 weeks after the operation. Downstream genes of Ihh signaling and OA-related gene expression were quantified by real-time polymerase chain reaction (PCR). The extent of OA progression was graded using Safranin-O and immunohistochemical staining. Ten rats were used to detect cartilage degeneration, and 15 rats were assessed using real-time PCR in each group.

\section{Histology}

Seventy-two hours after culture with ipriflavone or $0.1 \%$ DMSO, the human cartilage explants were fixed in $10 \%$ formalin (Sigma Aldrich, St Louis, MO, USA) for $72 \mathrm{~h}$. The specimens were decalcified in Richman-Gelfand-Hill 
solution, processed in a Tissue-Tek VIP 1000 tissue processor (Miles, Elkhart, IN, USA), and embedded in a single block of Paraplast X-tra (Thermo-Fisher, Hampton, NH, USA). Blocks were trimmed to expose the tissue using a rotary microtome (Leica RM2125, Leica Microsystems Ltd., Wetzlar, Germany). Ten adjacent sections were collected at intervals of $0 \mu \mathrm{m}, 100 \mu \mathrm{m}$, and $200 \mu \mathrm{m}$. Two serial $6-\mu \mathrm{m}$-thick sections from each interval were stained with Safranin O.

In the in vivo study, the femurs and tibiae were hemisected in the midsagittal plane, and each half was embedded in a single block of Paraplast X-tra (Thermo-Fisher, Hampton, NH, USA). Blocks were trimmed to expose the cartilage. Ten adjacent sections were collected at intervals of $0 \mu \mathrm{m}, 100 \mu \mathrm{m}$, and $200 \mu \mathrm{m}$ (Leica RM2125, Leica Microsystems Ltd., Wetzlar, Germany). Two serial 6- $\mu$ m-thick sections from each interval were stained with Safranin O. Cartilage degradation was quantified by two independent and blinded observers using an Osteoarthritis Research Society International (OARSI) grading system [27].

\section{Immunohistochemistry}

To detect the distribution of type II collagen, MMP-13, type $\mathrm{X}$ collagen, and type II collagen breakdown product (type II collagen-C fragment) in cartilage, we carried out immunohistochemistry using the 3,3'-diaminobenzidine (DAB) Histostain streptavidin-peroxidase (SP) kit (Novex, Life Technologies, USA). The sections $(6 \mu \mathrm{m})$ were deparaffinized and rehydrated using conventional methods. The sections were digested with $5 \mathrm{mg} / \mathrm{ml}$ of hyaluronidase in phosphate-buffered saline (PBS; Sigma) at $37^{\circ} \mathrm{C}$ for $10 \mathrm{~min}$. Endogenous peroxidase was blocked by treating the sections with $3 \%$ hydrogen peroxide in methanol (Sigma-Aldrich) at room temperature for 10 min. Nonspecific protein binding was blocked by incubation with a serum blocking solution (Li-Cor) at room temperature for $10 \mathrm{~min}$. The sections were incubated with specific antibodies against rat type II collagen, MMP-13, types X collagen, or type II collagen-C fragment (IBEX Technologies, Mont-Royal, QC, Canada) at $4{ }^{\circ} \mathrm{C}$ overnight. Thereafter, the sections were treated sequentially with biotinylated secondary antibody and SP conjugates at $37^{\circ} \mathrm{C}$ for $10 \mathrm{~min}$ and then developed in $\mathrm{DAB}$ chromogen (Invitrogen) for $3 \mathrm{~min}$. The sections were counterstained with hematoxylin (Invitrogen) for 1 min. Photomicrographs were obtained with a Nikon E800 microscope (Nikon, Melville, NY, USA).

\section{Western blotting}

The total protein of chondrocytes and cartilage explants was isolated and quantified using the BAC Protein Assay Reagent Kit (Pierce, Rockford, IL, USA). Fifteen micrograms of total protein was electrophoresed by $10 \%$ SDS
PAGE under reducing conditions. After electrophoresis, the proteins were transferred onto an $\mathrm{NC}$ membrane (Beyotime Biotechnology, China) and probed with a polyclonal antibody against Smo (SC-166685, Santa Cruz, CA, USA), type II collagen, MMP-13 (SC-52658, SC-515284 Santa Cruz, CA, USA), type X collagen (ab49945 Abcam, USA), and Runx-2 (SC-390351 Santa Cruz, CA, USA). The antibody was diluted 1:500 in PBS-T containing 1\% bovine serum albumin (BSA) (Sigma-Aldrich, St Louis, MO, USA). Horseradish peroxidase-conjugated secondary antibody IgG $(\mathrm{H}+\mathrm{L})$ (SC-2371, Santa Cruz, CA, USA) was diluted 1:2000 in PBS-T. Visualization of immunoreactive proteins was achieved using ECL Western blotting detection reagents (KWBIO, Beijing, China) and subsequently exposing the membrane to Kodak X-Omat AR film (Kodak, Rochester, NY). Band densities were quantified using Image Acquisition and Analysis Software (UVP, Upland, CA, USA).

\section{Real-time quantitative PCR}

The total RNA was isolated from human chondrocytes and rat knee joint cartilage using an RNeasy isolation kit (Takara, Japan). Cartilage samples (tibial plateau and femur condyle) from three rats were dissected using a scalpel and pooled together; there were 5 pooled samples per group. Total RNA $(1 \mu \mathrm{g})$ was reverse-transcribed to complementary DNA (cDNA) using a reverse transcription kit (Takara, Japan). The resulting cDNA $(80 \mathrm{ng} / \mu \mathrm{l})$ was used as the template to quantify the relative content of messenger RNA (mRNA) using a QuantiTect SYBR Green PCR kit (Takara, Japan) with an IQ5 Fluorescence Detection System (Bio-Rad). Primer pairs were listed in Table 1. Relative transcript levels were calculated according to the equation $x=2^{-\Delta \Delta \mathrm{Ct}}$, where $\Delta \Delta \mathrm{C}_{\mathrm{t}}=\Delta \mathrm{C}_{\mathrm{t}} \mathrm{E}-\Delta \mathrm{C}_{\mathrm{t}}$ $\mathrm{C}\left(\Delta \mathrm{C}_{\mathrm{t}} \mathrm{E}=\mathrm{C}_{\mathrm{t}} \exp -\mathrm{C}_{\mathrm{t}} 18 \mathrm{~S}\right.$ and $\left.\Delta \mathrm{C}_{\mathrm{t}} \mathrm{C}=\mathrm{C}_{\mathrm{t}} \mathrm{C}-\mathrm{C}_{\mathrm{t}} 18 \mathrm{~S}\right)$ [6].

\section{Cell proliferation assays}

Cell proliferation was detected using the Cell Counting Kit-8 (CCK-8) cell viability assay kit (Boster Biological Technology, China) and Cell-Light ${ }^{\mathrm{tm}}$ EdU Kit (RiboBio, Guangzhou, China) according to the manufacturer's protocol. The CCK- 8 assay was performed to detect the proliferation of chondrocytes treated with ipriflavone for 1 day, 2 days, 3 days, and 4 days. The cell suspension $(100 \mu \mathrm{l} /$ well $)$ was added to a 96 -well plate, and the plate was preincubated in a humidified incubator (at $37^{\circ} \mathrm{C}, 5 \%$ $\mathrm{CO}_{2}$ ). Next, $10 \mu \mathrm{l}$ of the CCK-8 solution was added to each well of the plate and incubated for $4 \mathrm{~h}$ in the incubator, followed by measuring the absorbance at $450 \mathrm{~nm}$ using microplate reader (Bio-Rad, CA, USA). EdU was added to the culture medium at a concentration of $50 \mu \mathrm{M}$. The cells were fixed with $4 \%$ paraformaldehyde, permeabilized with $0.5 \%$ Triton X-100 in PBS for 15 min, and subsequently incubated with Apollo ${ }^{\circ}$ reaction 
Table 1 Primers used in this paper with their species, name, orientation, and sequence used in the RT-PCR protocol

\begin{tabular}{|c|c|c|c|}
\hline Species & Name & Forward/reverse & Sequence \\
\hline \multirow[t]{20}{*}{ Human } & Col2a1 & Forward & TGA GGG CGC GGT AGA GAC CC \\
\hline & Col2a1 & Reverse & TGC ACA CAG CTG CCA GCC TC \\
\hline & Col10a1 & Forward & TGC CTC TTG TCA GTG CTA ACC \\
\hline & Col10a1 & Reverse & GCG TGC CGT TCT TAT ACA GG \\
\hline & MMP-13 & Forward & TGC TGC ATT CTC CTT CAG GA \\
\hline & MMP-13 & Reverse & ATG CAT CCA GGG GTC CTG GC \\
\hline & Runx-2 & Forward & GGC AGG CAC AGT CTT CCC \\
\hline & Runx-2 & Reverse & GGC CCA GTT CTG AAG CAC C \\
\hline & Smo & Forward & CCT TTG GCT TTG TGC TCA TTA CCT T \\
\hline & Smo & Reverse & CGT CAC TCT GCC CAG TCA ACC T \\
\hline & Gli1 & Forward & GAA CCC TTG GAA GGT GAT ATG TC \\
\hline & Gli1 & Reverse & GGC AGT CAG TTT CAT ACA CAG AT \\
\hline & Gli2 & Forward & GCG TGT TTA CCC AAT CCT GT \\
\hline & Gli2 & Reverse & GAT GCT CCC TCA GAG TCC TG \\
\hline & Hhip & Forward & TCC GGT CAC ATC TTG GGA TT \\
\hline & Hhip & Reverse & GTC TGT GCA GGT TGT ACC GTG \\
\hline & Ptc1 & Forward & ATG CTG GCG GGA TCT GAG TTC GAC T \\
\hline & Ptc1 & Reverse & GGG TGT GGG CAG GCG GTT CAA G \\
\hline & Gli3 & Forward & CTT TGC AAG CCA GGA GAA AC \\
\hline & Gli3 & Reverse & TTG TTG GAC TGT GTG CCA TT \\
\hline \multirow[t]{16}{*}{ Rat } & $\mathrm{Col} 2$ & Forward & GAG GGC AAC AGC AGG TTC AC \\
\hline & Col2 & Reverse & TGT GAT CGG TAC TCG ATG ATG G \\
\hline & Agg & Forward & CAG TGC GAT GCA GGC TGG CT \\
\hline & Agg & Reverse & CCT CCG GCA CTC GTT GGC TG \\
\hline & Col10 & Forward & CCA GGT GTC CCA GGA TTC CC \\
\hline & Col10 & Reverse & CAA GCG GCA TCC CAG AAA GC \\
\hline & MMP13 & Forward & GGA CCT TCT GGT CTT CTG GC \\
\hline & MMP13 & Reverse & GGA TGC TTA GGG TTG GGG TC \\
\hline & Smo & Forward & TCT CGG GCA AGA CAT CCT \\
\hline & Smo & Reverse & TAG CCT CCC ACA ATA AGC A \\
\hline & Gli-1 & Forward & GCC AAT CAC AAA TCA GTC TCC \\
\hline & Gli-1 & Reverse & TGC TCC TAA CCT GCC CAC \\
\hline & Gli-2 & Forward & AGG CCC AGT ACA TGC TGG TTG \\
\hline & Gli-2 & Reverse & GGA CCG CAG GTG TGT CTT CA \\
\hline & Gli-3 & Forward & TGG GAT TCC GAC GGT TCT G \\
\hline & Gli-3 & Reverse & GGA GGT CTT CAT CGG GCT TG \\
\hline \multirow[t]{2}{*}{ Human and rat } & $18 S$ & Forward & CGG CTA CCA CAT CCA AGG AA \\
\hline & 185 & Reverse & GCT GGA ATT ACC GCG GCT \\
\hline
\end{tabular}

cocktail (containing Apollo $^{\circ}$ reaction buffer, Apollo ${ }^{\circ}$ catalyst, Apollo 567 fluorescent dyes and buffer additives) and Hoechst 33342, for $30 \mathrm{~min}$ away from light. The cells were then observed immediately under a fluorescence microscope (Olympus, Japan). The percentage of positive cells (red) was determined.

\section{Cell apoptosis assay by flow cytometry}

Cell apoptosis was detected using a Cell Apoptosis Assay Kit (BOSTER Biological Technology, China) according to the manufacturer's protocol. After $48 \mathrm{~h}$ in culture with ipriflavone or DMSO, chondrocytes were digested with $0.25 \%$ trypsin and dissociated into single cells, followed 
by double-staining with fluorescein FITC-labeled annexin $\mathrm{V}$ and propidium iodide (PI) in binding buffer (BD, USA) for 15 min away from light. Finally, apoptosis was detected by flow cytometry (FCM, BD, USA). The experiment was repeated three times. Cells that were positive for annexin $\mathrm{V}$ alone were recognized as early apoptosis, cells that were positive for PI only were recognized as necrosis, and cells that were positive for both annexin V and PI were recognized as late apoptosis.

\section{Statistical analysis}

The data represent the means $\pm \mathrm{SD}$ obtained from at least three independent experiments. Each experimental measure was performed in triplicate. Two-tailed paired $t$ tests were used to compare the changes in gene expression levels and protein expression levels of Smo, Gli-1, $-2,-3$, Runx-2, type II collagen, type $\mathrm{X}$ collagen, and MMP-13. $P$ values less than 0.05 were considered significant. Statistical analyses were performed using SPSS software.

\section{Results \\ Ipriflavone promoted the proliferation and reduced the apoptosis of human chondrocytes}

To determine the effect of ipriflavone on the proliferation of chondrocytes, an EdU-based cell proliferation assay and CCK- 8 assay were performed. As revealed by EdU cell proliferation staining (red) (Fig. 1B-a), ipriflavone significantly promoted chondrocyte proliferation. Approximately 300 cells from 3 independent experiments were scored, and the percentages of EdU-positive cells in the $5 \mu \mathrm{M}$ and $10 \mu \mathrm{M}$ ipriflavone groups were $36.40 \% \pm 1.32 \%$ and $28.54 \% \pm 0.74 \%$, respectively $(P<$ 0.001 ), whereas human OA chondrocytes treated with DMSO had the lowest EdU-positive stained cells $(11.99 \% \pm 0.35 \%)$ (Fig. 1B-b). The CCK-8 assay results showed that the viability of chondrocytes was higher in the ipriflavone treatment groups than the DMSO control group, and the viability gradually increased with a longer treatment time (Fig. 1B-c). To further determine the effect of ipriflavone on chondrocyte apoptosis, we performed an annexin V-FITC/propidium iodide (PI) dual staining assay by flow cytometry, and the results showed that apoptosis was reduced in the ipriflavone treatment group than the DMSO control group after $48 \mathrm{~h}$ of treatment (Fig. 1C-a). To confirm the above results, the cellular apoptosis rate was measured. The results demonstrated that the percentage of apoptotic cells in the DMSO, $5 \mu \mathrm{M}$ or $10 \mu \mathrm{M}$ ipriflavone groups was $25.76 \% \pm 5.1 \%, 12.64 \% \pm 3.7 \%$, and $15.18 \% \pm 3.13 \%$, respectively $(P<0.05)$ (Fig. 1C-b). These findings suggested that ipriflavone was able to increase the proliferation and decrease the apoptosis of chondrocytes in vitro.
Ipriflavone downregulated OA-related gene and protein expression in human chondrocyte culture by inhibiting Ihh signaling

The results of real-time PCR indicated that ipriflavone significantly decreased the mRNA levels of key genes in the Ihh signal pathway (Smo, Gli2, Runx-2) at both $5 \mu \mathrm{M}$ and $10 \mu \mathrm{M}$ after $48 \mathrm{~h}$ of treatment; however, the mRNA levels of Gli1 and Gli3 were decreased only in the $10 \mu \mathrm{M}$ ipriflavone treatment group. Ipriflavone also decreased the expression of MMP-13 and type X collagen mRNA and increased the expression of type II collagen mRNA in both ipriflavone groups (Fig. 2A). The Western blotting results showed that compared with the DMSO control group, the expression of key proteins in Ihh signaling (Smo and Runx-2) were significantly decreased in both the $5 \mu \mathrm{M}$ and $10 \mu \mathrm{M}$ ipriflavone treatment groups after $48 \mathrm{~h}$, and the expression of MMP-13 and type $\mathrm{X}$ collagen was also significantly decreased at both concentrations. Simultaneously, the expression of type II collagen was significantly increased (Fig. 2B). These results suggested that ipriflavone had a chondroprotective effect by decreasing OA-related gene and protein expression and increasing the expression of anabolic factors by inhibiting the Ihh pathway.

\section{Ipriflavone reduced the degeneration of cartilage by inhibiting lhh signaling in cultured human cartilage explants}

To confirm the results from the monocultures, human cartilage explants $\left(4 \mathrm{~mm}^{3}\right.$ pieces $)$ were treated with $50 \mu \mathrm{M}$ ipriflavone, $100 \mu \mathrm{M}$ ipriflavone, and DMSO. After $72 \mathrm{~h}$ in culture without removing the reagent, the total mRNA and total protein were isolated from the cartilage tissue to detect the expression of key genes and proteins, respectively. Real-time PCR results showed that the mRNA levels of Smo, Gli-2, and Runx-2 were decreased in both ipriflavone treatment groups. Type II collagen mRNA levels were increased while MMP-13 and type X collagen mRNA levels were decreased in both ipriflavone treatment groups (Fig. 3B). Western blotting results indicated that the expression of key proteins in Ihh signaling (Smo and Runx-2 protein) were decreased in both ipriflavone treatment groups compared with the DMSO control group. MMP-13 and type $\mathrm{X}$ collagen proteins were also reduced, while the expression of type II collagen protein was higher than in the DMSO control group (Fig. 3C).

\section{Ipriflavone attenuated the degeneration of articular cartilage by blocking the Indian hedgehog pathway in rat OA models}

To further test ipriflavone in vivo, it was intragastrically administered in the rat ACLT model of OA $(200 \mathrm{mg} / \mathrm{kg})$. Cartilage degeneration was analyzed after 12 weeks of 

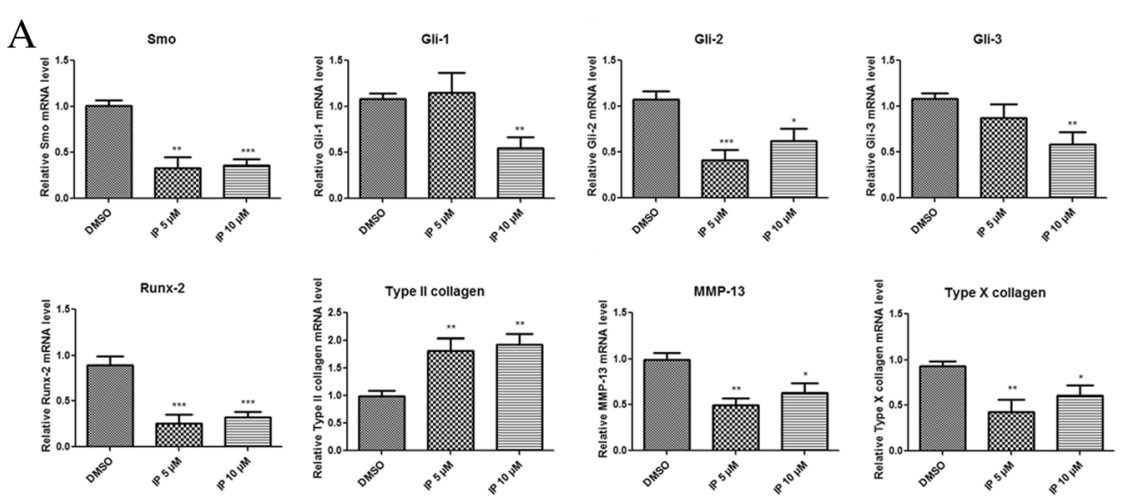

B
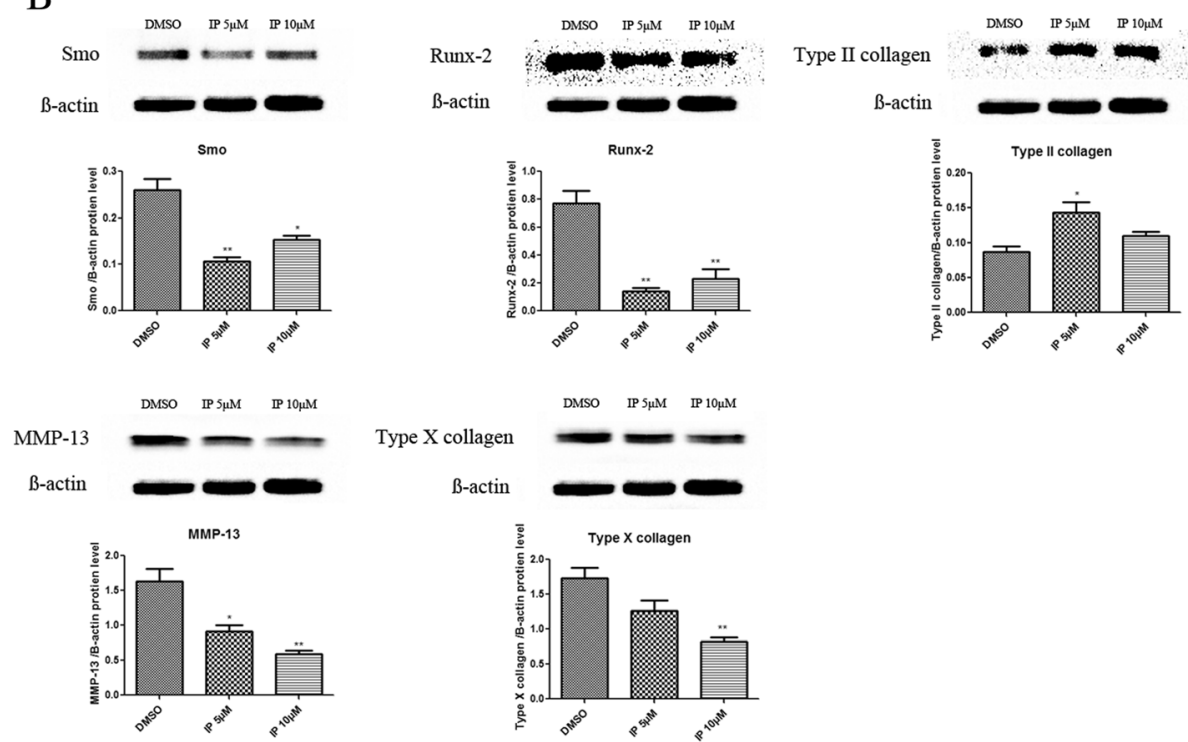

Fig. 2 Chondroprotective effect of ipriflavone (IP) in human chondrocytes. a Real-time PCR results showed reduced mRNA expression of key genes in the Ihh pathway, Smo, Gli-1,Gli-2,Gli-3, and Runx-2, at $48 \mathrm{~h}$ after IP treatment, and among the three kinds of Glis, the reduction of Gli-2 was especially significant. The MMP-13 and type X collagen mRNA levels were decreased, and the type II collagen mRNA level was significantly increased in human chondrocytes. $\mathbf{b}$ Western blot results indicated that in chondrocytes, the expression of Smo and Runx-2 protein was decreased at $48 \mathrm{~h}$ after IP treatment, MMP-13 and type X collagen expression was decreased in the IP treatment group, and type II collagen expression was increased in the IP treatment group. The gray value of the Western blot bands was semiquantified using Image Analysis Software (Image Lab 3.0). Values are the mean \pm SEM. $n=3,{ }^{*} P<0.05,{ }^{*} P<0.01,{ }^{* *} P<0.001$ versus the DMSO group

treatment by histology. Safranin O staining demonstrated that there was less surface damage with stronger Safranin O staining in articular cartilage specimens from ipriflavone-treated animals compared with ACLT group animals. Osteoarthritis scores (OARSI scores) were significantly reduced in the ipriflavone treatment group compared with the ACLT group (Fig. 4A). The results of immunohistochemical staining indicated that type II collagen expression in articular cartilage was higher in the ipriflavone-treated and sham-operated rats than in rats that underwent ACLT without treatment. In contrast, degraded collagen II (type II collagen-C fragment), type $\mathrm{X}$ collagen, and matrix metalloproteinase 13 (MMP-13) staining were elevated in rats that underwent ACLT without treatment with respect to the ipriflavone-treated and sham-operated rats (Fig. 4B), which is consistent with the reduced OA damage. Real-time PCR results indicated that the expressions of key genes in the Ihh pathway (Smo, Gli-1, Gli-2, Gli-3) were increased in rats that underwent ACLT without IP treatment, and they were reduced in the ipriflavone-treated rats at12 weeks after the ACLT operation, especially Smo and Gli-2. Simultaneously, type II collagen and Agg expression in articular cartilage was higher in the ipriflavone-treated and sham-operated rats than in rats that underwent ACLT without treatment. In contrast, type $X$ collagen and MMP-13 were elevated in rats that underwent ACLT without ipriflavone treatment, and the expression levels were reduced in ipriflavone-treated rats at 12 weeks after the ACLT operation (Fig. 4C), which is 

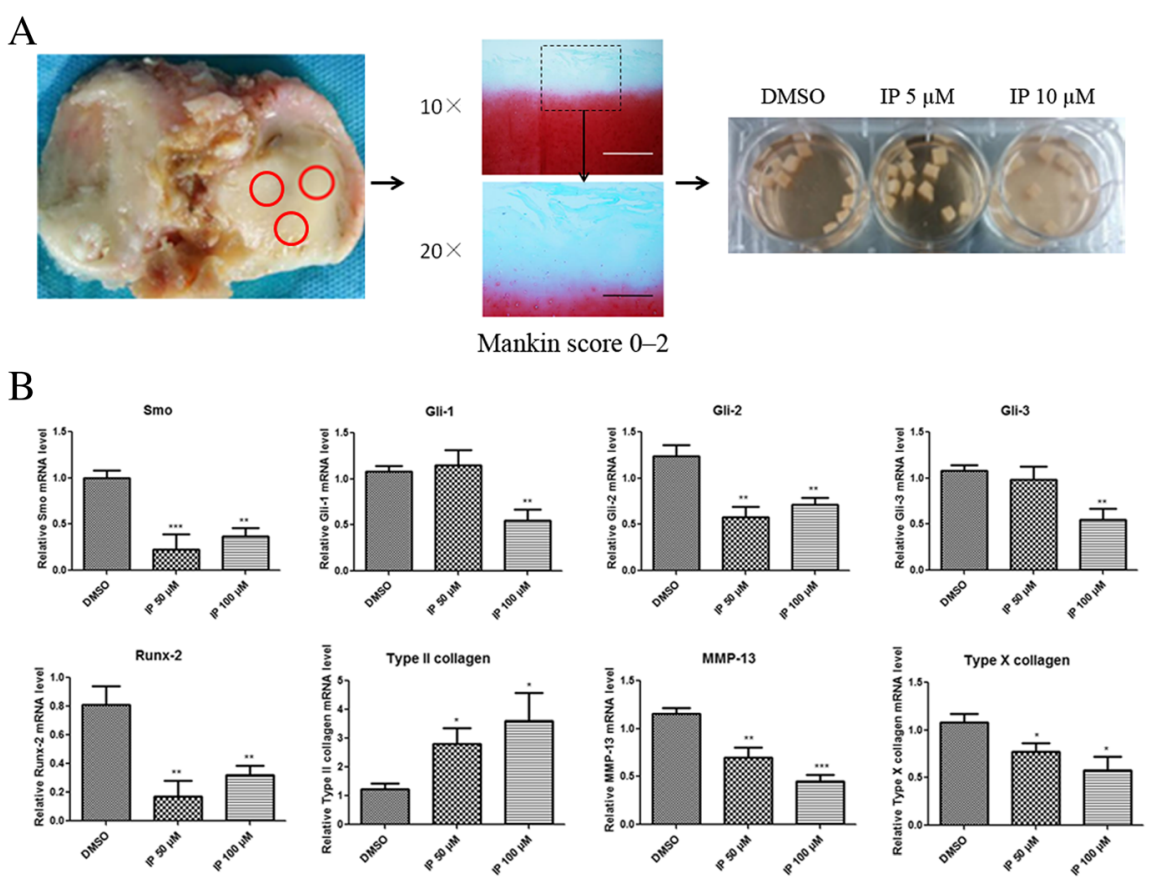

C

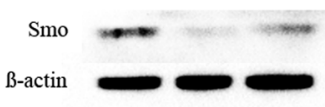

smo

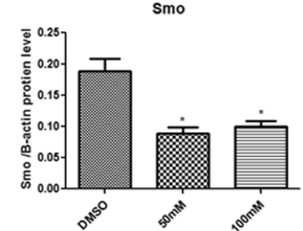

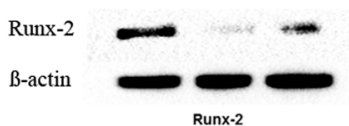

Runx-2

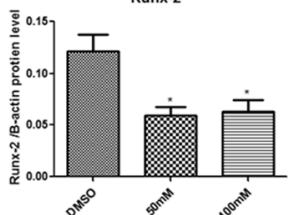

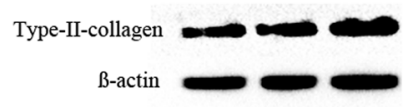

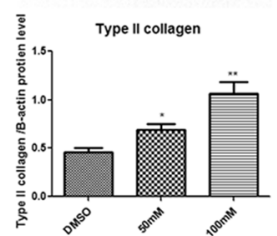

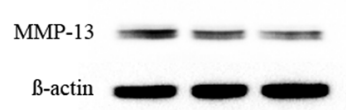

MMP-13

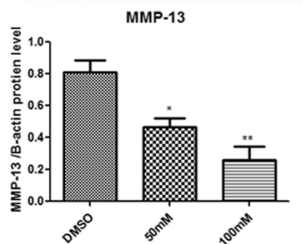

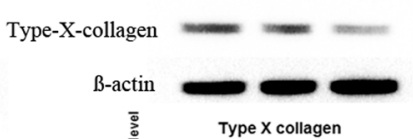

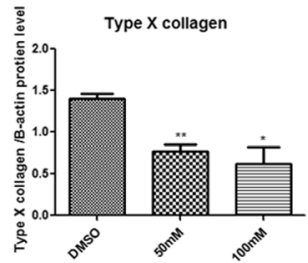

Fig. 3 Ipriflavone reduced the degeneration of cartilage by inhibiting Ihh signaling in cultured human cartilage explants. a Safranin $O$ staining results showed that cartilage samples were taken from "relatively normal" cartilage samples of the tibia obtained during total knee arthroplasty (Mankin score 0-2). Then, the cartilage samples were cut into 4- $\mathrm{mm}^{3}$ pieces weighing 6-9 mg each and randomly subdivided into three groups: $0.1 \%$ DMSO group, $50 \mu \mathrm{M}$ IP treatment group, and $100 \mu \mathrm{M}$ IP treatment group. $\mathbf{b}$ Real-time PCR results showed that the mRNA expression of key genes in Ihh pathway, Smo, Gli-1,Gli-2,Gli-3, and Runx-2 was decreased in the IP treatment group, especially the reduction of Gli-2.

Simultaneously, the mRNA levels of type II collagen was increased while MMP-13 and type X collagen were decreased in both IP treatment groups. c Western blotting results indicated that the expression of key proteins in Ihh signaling (Smo and Runx-2) were decreased in the IP treatment groups compared with the DMSO control group. MMP-13 and type X collagen protein were also reduced, while the expression of type II collagen protein was increased compared with the DMSO control group. Values are the mean \pm SEM. $n=3$, * $P<0.05,{ }^{* *} P<0.01$ versus the DMSO and IP treatment group 

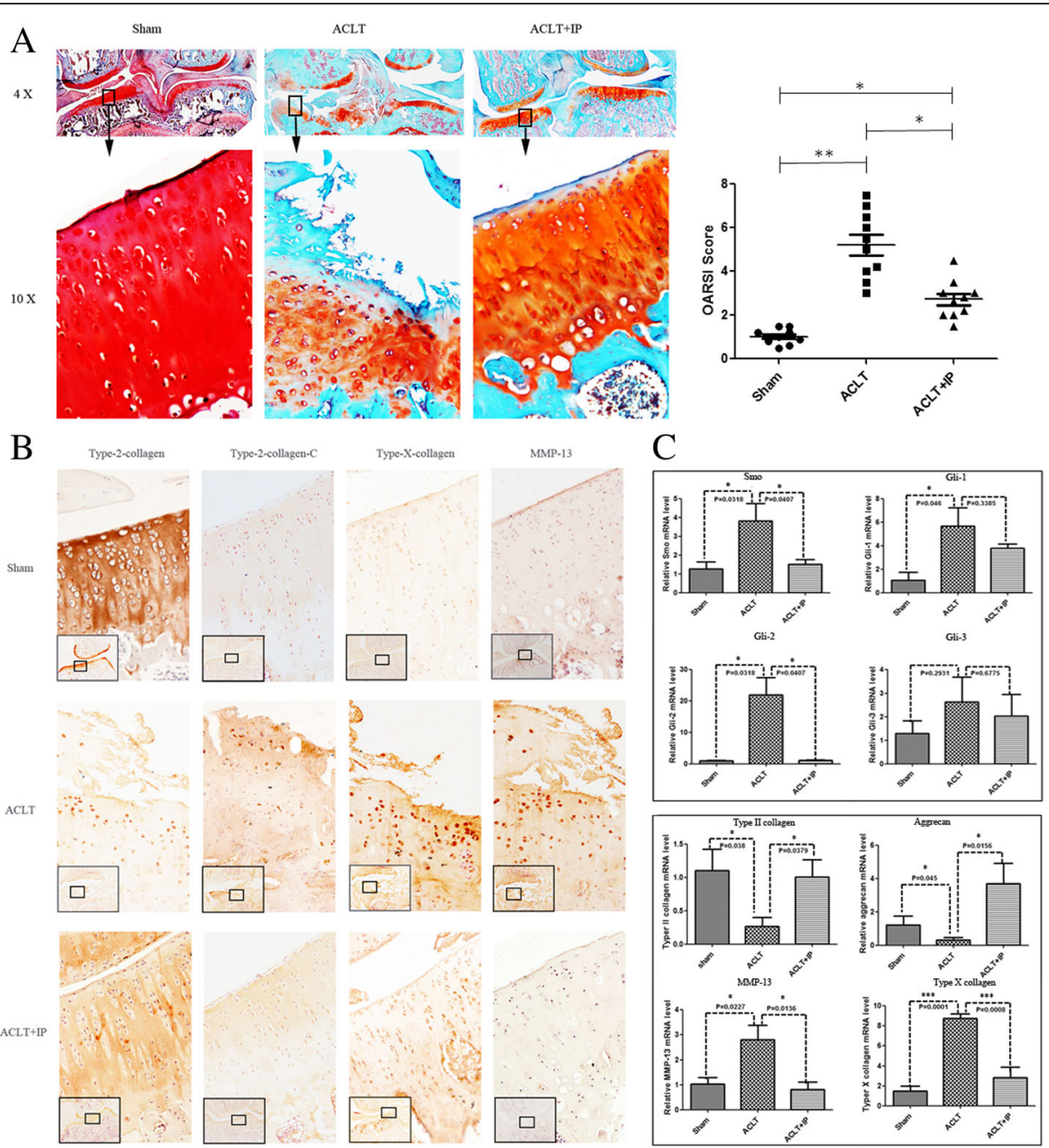

Fig. 4 Ipriflavone (IP) reduced the degeneration of cartilage by blocking the Indian hedgehog pathway in vivo. a The results of Safranin $\mathrm{O}$ staining indicated that there was less surface damage with stronger Safranin O staining in articular cartilage specimens from IP-treated animals compared with the ACLT group at 12 weeks after the operation. Cartilage destruction was quantified and compared with the ACLT group, and the summed OARSI scores were significantly decreased in the IP treatment group. Data are expressed as means \pm SDs. $(n=10),{ }^{*} P<0.05{ }^{* *} P<$ 0.01. b Type II collagen expression in articular cartilage was higher in IP-treated and sham-operated rats than in rats that underwent ACLT without treatment. In contrast, degraded collagen II (type II collagen-C fragment), type X collagen, and matrix metalloproteinase 13 (MMP-13) staining levels were elevated in rats that underwent ACLT without treatment with respect to the IP-treated and sham-operated rats, which is consistent with reduced OA damage. c Real-time PCR results indicated that the expression of key genes in the Ihh pathway (Smo, Gli-1, Gli-2, Gli-3) was increased in rats that underwent ACLT without IP treatment and reduced in IP-treated rats at 12 weeks after the ACLT operation, especially Smo and Gli-2. Concomitantly, type II collagen and Agg expression in articular cartilage was elevated in IP-treated and sham-operated rats compared with rats that underwent ACLT without treatment. In contrast, type X collagen and matrix metalloproteinase 13 (MMP-13) were elevated in rats that underwent ACLT without IP treatment, and their expressions were reduced in IP-treated rats at 12 weeks after the ACLT operation, consistent with the reduced OA damage. Values are the mean \pm SEM. $P<0.05$ versus the ACLT and IP treatment group

consistent with the reduction of OA damage by blocking Ihh signaling.

\section{Discussion}

Ihh is a secreted protein that is expressed in prehypertrophic chondrocytes [28]. This protein is highly important in the regulation of hypertrophic differentiation of articular chondrocytes during endochondral ossification $[29,30]$. Studies have shown that Ihh can bind Patched-1 (PTCH1) receptor, relieving Smoothened (Smo) inhibition and activating the glioma-associated oncogene homolog (GLI) family (Gli1/2/3), which will upregulate the gene expression of Runt-related transcription factor 2 (Runx-2) and promote chondrocyte hypertrophy [3-5].

Articular chondrocytes become hypertrophic in OA cartilage [31, 32]. The collective evidence indicates that Ihh plays a critical role in OA cartilage degeneration and that Ihh blockade may have a chondroprotective effect for OA treatment. However, Ihh inhibitors such as cyclopamine, the first Smo antagonist, cause serious side effects, 
including weight loss and dehydration [33, 34], holoprosencephaly (HPE), cleft lip palate (CLP), and limb defects in vivo [9-13]. To overcome these side effects, some modified cyclopamine prodrugs have been developed, such as IPI-269609 and IPI-926 [35, 36], but these drugs are only used as anticancer agents and still exhibit high toxicity to other tissues and organs, limiting the broad use of inhibitors of the Ihh pathway in humans. The results of our study demonstrate, for the first time, that ipriflavone, a novel and safe inhibitor of Ihh signaling, can attenuate cartilage degeneration by blocking the Ihh pathway in vitro and in vivo.

Our in vitro and in vivo data clearly demonstrated the following: (1) Ipriflavone promoted the proliferation and reduced the apoptosis of human chondrocytes. (2) Ipriflavone inhibited the expression of target gene Runx-2 by Smo-Gli2 pathway mainly. (3) The expression of OA-related genes and proteins, including MMP-13 and type $\mathrm{X}$ collagen, were reduced while type II collagen was increased after ipriflavone treatment in vitro and in vivo, indicating that ipriflavone is a promising therapeutic drug to inhibit cartilage degeneration by blocking Ihh signaling. Our results are in agreement with our previous study [16] and the findings of others [10], which reported that inhibition of Ihh signaling has the potential to prevent surgically induced $\mathrm{OA}$ in transgenic mice. These findings suggest that ipriflavone may be a safe and effective oral medicine for the treatment of OA.

Dose-response assays of ipriflavone in a previous study suggested that concentrations of ipriflavone between $1 \mu \mathrm{M}$ and $10 \mu \mathrm{M}$ can inhibit the Ihh signaling pathway, and the inhibition rates of $1 \mu \mathrm{M}$ and $10 \mu \mathrm{M}$ ipriflavone were approximately $20 \%$ and $100 \%$, respectively [14]. In our pilot study, we treated chondrocytes with $1 \mu \mathrm{M}, 2.5 \mu \mathrm{M}, 5 \mu \mathrm{M}$, and $10 \mu \mathrm{M}$ ipriflavone. The results showed that the gene expression of Smo, the key downstream gene of the Ihh pathway, was blocked significantly in the $5 \mu \mathrm{M}$ and $10 \mu \mathrm{M}$ groups (Additional file 1: Figure S1). We further measured the proliferation and apoptosis of the chondrocytes in the $5 \mu \mathrm{M}$ and $10 \mu \mathrm{M}$ groups, and the results indicated significantly increased proliferation and decreased apoptosis in the two ipriflavone treatment groups, which demonstrated that these two doses are safe in human chondrocytes. Interestingly, upon treating human chondrocytes with ipriflavone and Ihh recombinant protein $(5.0 \mu \mathrm{g} / \mathrm{ml}[6])$, we observed a change in proliferation and apoptosis (Additional file 2: Figure S2), which indicated that the changes in cell proliferation and apoptosis induced by ipriflavone may be regulated by the inhibition of the Ihh signaling pathway. Promoting the proliferation of and inhibiting apoptosis of chondrocytes may be one of the mechanisms of the treatment of OA with ipriflavone.

A previous study reported that ipriflavone acts downstream of Ptc1 and upstream of Gli and that it acts similarly to cyclopamine [14]. However, the function of Gli1/2/3 in this process is still incompletely understood. Some studies have shown that Gli2 and Gli3 are essential for skeletal development, whereas Gli1 is not critical to this process; Gli1 acts synergistically with Gli2 and Gli3 [37]. Other studies have demonstrated that Gli1 and Gli2 seem to act mainly as activators of hedgehog target genes in the Ihh signaling pathway, and Gli3 may function both as a transcription factor and as a repressor of Ihh target genes [38, 39]. Our in vitro results indicated that only Gli2 was decreased in both IP groups, and our in vivo experiments showed similar results, specifically that only Gli2 was inhibited significantly; these findings suggest that ipriflavone inhibits Runx-2 mainly through the Smo-Gli2 pathway. In addition, we detected the mRNA expression of other relative genes of Ihh signaling, the results further confirmed our results too (Additional file 3: Figure S3). Our results are consistent with other reports [40].

The limitation of this study is that surgical transection of the ACL may not be as traumatic as an ACL injury sustained during physical activity. Bone bruises and chondral lesions frequently occur in the latter, and these concomitant injuries may also play a role in the development of posttraumatic OA. Nonetheless, the animal model of ACLT has been frequently used to study OA, and it mimics human OA both macroscopically and biochemically [41]. Minimizing local joint inflammation until ACL reconstruction is performed may be an important preventive measure that could forestall the long-term development of posttraumatic OA (Additional file 3).

\section{Conclusions}

This study provides direct evidence that ipriflavone is able to attenuate cartilage degeneration by inhibiting Ihh signaling. Thus, ipriflavone could be a potential chondroprotective drug for OA treatment.

\section{Additional files}

Additional file 1: Real-time PCR results showed reduced mRNA expression of key genes in the Ihh pathway, Smo, at $48 \mathrm{~h}$ after $5 \mu \mathrm{M}$ and $10 \mu \mathrm{M}$ groups but not in the cells treated with the $1 \mu \mathrm{M}$ and $2.5 \mu \mathrm{M}$ concentration. Values are the mean \pm SEM. $n=3,{ }^{* *} p<0.01$, versus the DMSO group. (TIF $117 \mathrm{~kb}$ )

Additional file 2: Human chondrocyte treated with ipriflavone and Ihh recombinant protein did change the level of the cell proliferation and apoptosis significantly. Primary chondrocytes were incubated in DMEM containing $10 \% \mathrm{FBS}$, under $37^{\circ} \mathrm{C}, 5 \% \mathrm{CO}_{2}$ condition. The cells were treated with $5 \mu \mathrm{M}$ ipriflavone, $5 \mu \mathrm{M}$ ipriflavone +lhh $(5.0 \mu \mathrm{g} / \mathrm{ml})$ recombinant protein, respectively, and the $0.1 \%$ DMSO treatment group was used as the control. After $48 \mathrm{~h}$ in culture without removing the reagent, the cell proliferation assay and cell apoptosis assay were performed. A-a The EdU-based cell proliferation assay showed that compared with the DMSO group, the EdU-positive cells (red) were significantly increased in the $5 \mu \mathrm{M} I \mathrm{P}$ treatment group, and compared with the $5 \mu \mathrm{M}$ IP treatment group, it was decreased in the $5 \mu \mathrm{M} I \mathrm{P}+\mathrm{Ihh}$ 
treatment group significantly. A-b The percentage of EdU-positive cells was quantified, Data are expressed as means \pm SDs $(n=3)^{* * *} P<0.001$ versus the DMSO group, ${ }^{* *} P<0.01$ versus the $5 \mu \mathrm{M}$ IP treatment group. A-c The CCK-8 assay results showed that the viability of chondrocytes was higher in $5 \mu \mathrm{M} I \mathrm{P}$ treatment group than the DMSO control group, and the viability of chondrocytes was decreased by Ihh treatment. Data are expressed as means \pm SDs $(n=3){ }^{* *} P<0.01,{ }^{*} P<0.05$ versus the DMSO group. B-a The Annexin V-FITC/propidium iodide (PI) dual staining assay by flow cytometry indicated that apoptosis was reduced in the $5 \mu \mathrm{M} \mathrm{IP}$ treatment group compared with the DMSO control group at $48 \mathrm{~h}$ after treatment, and the reduction was blocked by Ihh treatment. Values are the mean \pm SDs. $(n=3){ }^{*} P<0.05$ versus $5 \mu M$ IP treatment group. (TIF $\left.3873 \mathrm{~kb}\right)$

Additional file 3: Real-time PCR results showed reduced mRNA expression of key genes in the Ihh pathway, Ptch1, and Hhip, at $48 \mathrm{~h}$ after $5 \mu \mathrm{M}$ and $10 \mu \mathrm{M}$ groups. Values are the mean \pm SEM. $n=3,{ }^{*} P<0.05$ versus the DMSO group. (TIF $161 \mathrm{~kb}$ )

\section{Abbreviations}

ACLT: Anterior cruciate ligament transection; Agg: Aggrecanase; BSA: Bovine serum albumin; CCK-8: Cell Counting Kit-8; DAB: 3,3'-Diaminobenzidine; DMEM: Dulbecco's modified Eagle's medium; DMSO: Dimethyl sulfoxide: FBS: Fetal bovine serum; Gli: Glioma-associated oncogene homolog; HBSS: Hank's Balanced Salt Solution; Hh: Hedgehog; Ihh: Indian hedgehog; IP: Ipriflavone; MMP-13: Matrix metallopeptidase 13; OA: Osteoarthritis; OARSI: Osteoarthritis Research Society International; PBS: Phosphate-buffered saline; PTCH1: Patched-1; PTOA: Posttraumatic osteoarthritis; Runx-2: Runtrelated transcription factor 2; Smo: Smoothened; SP: Streptavidin peroxidase

\section{Acknowledgements}

The content is solely the responsibility of the authors and does not necessarily represent the official view of the National Institutes of Health. The authors gratefully acknowledge all authors of this article, all funding supporting the project and American Journal Experts (AJE) for help with the manuscript preparation and editorial services.

\section{Funding}

The project was supported by Grant NSFC 81572098, 81772415, 81772867, SXNSF 2014021039-1, and ISTCP 2015DFA33050.

\section{Availability of data and materials}

All data generated or analyzed during this study are included in this published article.

\section{Authors' contributions}

LG participated in the design of the study and drafted, the part of in vitro and in vivo experiments, and revised the manuscript. XCW conceived the study, revised the manuscript, and performed the statistical analysis. ZWZ prepared the cartilage specimens and completed the part of in vitro experiments. XJW carried out the molecular genetics studies and performed the statistical analysis. CLW carried out the part of in vivo experiments and helped to perform the statistical analysis. PCL helped to revise the manuscript and performed the statistical analysis. CFW provided the lab for feeding the rats. LW conceived the study, participated in its design and coordination, and helped to draft the manuscript. All authors read and approved the final manuscript.

\section{Ethics approval and consent to participate}

Human chondrocyte and cartilage explants used in this study were approved by the Institutional Review Board at the second Hospital of Shanxi Medical University (Taiyuan, China; CMTT\#: 2013025. Approval 3 June 2013), and the patients agreed to participate in the research. The animal experiments were from Department of the Experimental Animal Center, Shanxi Medical University. All animal experiments were approved by the Institutional Review Board at the Second Hospital of Shanxi Medical University (Taiyuan, China; CMTT\#: 2013012. Approval 2013).

\section{Competing interests}

The authors declare that they have no competing interests.

\section{Publisher's Note}

Springer Nature remains neutral with regard to jurisdictional claims in published maps and institutional affiliations.

\section{Author details}

${ }^{1}$ Department of Orthopedics, Second Hospital of Shanxi Medical University, Shanxi Key Laboratory of Bone and Soft Tissue Injury Repair, Taiyuan, China No. 382, Wuyi Road, Taiyuan 030001, China. ${ }^{2}$ Shanxi Key Laboratory of Laboratory Animal and Animal Model of Human Diseases, Department of Experimental Animal Center, Shanxi Medical University, No. 56, Xinjian Southern Road, Taiyuan 030001, China. ${ }^{3}$ Department of Orthopedics, Warren Alpert Medical School of Brown University, Suite 402A, 1 Hoppin Street, Providence, RI 02903, USA.

Received: 24 October 2018 Accepted: 11 April 2019

Published online: 02 May 2019

\section{References}

1. Emery CA, Roos EM, Verhagen E, Finch CF, Bennell KL, Story B, Spindler K, Kemp J, Lohmander LS. OARSI clinical trials recommendations: design and conduct of clinical trials for primary prevention of osteoarthritis by joint injury prevention in sport and recreation. Osteoarthr Cartil 2015; 23(5): 815-5.

2. Lin AC, Seeto BL, Bartoszko JM, Khoury MA, Whetstone H, Ho L, Hsu C, Ali $\mathrm{SA}$, Alman BA. Modulating hedgehog signaling can attenuate the severity of osteoarthritis. Nat Med. 2009;15(12):1421-5.

3. Wang W, Lian N, Li L, Moss HE, Wang W, Perrien DS, Elefteriou F, Yang X. Atf4 regulates chondrocyte proliferation and differentiation during endochondral ossification by activating Ihh transcription. Development. 2009;136(24):4143-53.

4. Maeda Y, Nakamura E, Nguyen M-T, Suva LJ, Swain FL, Razzaque MS, Mackem S, Lanske B. Indian hedgehog produced by postnatal chondrocytes is essential for maintaining a growth plate andtrabecular bone. Proc Nat Acad Sci U S A. 2007;104(15):6382-7.

5. Mak KK, Kronenberg HM, Chuang P-T, Mackem S, Yang Y. Indian hedgehog signals independently of PTHrP to promote chon- drocyte hypertrophy. Development. 2008;135(11):1947-56.

6. Wei F, Zhou J, Wei X, Zhang J, Fleming BC, Terek R, Pei M, Chen Q, Liu T, Wei L. Activation of Indian hedgehog promotes chondrocyte hypertrophy and upregulation of MMP-13 in human osteoarthritic cartilage. Osteoarthr Cartil. 2012;20(7):755-63.

7. Zhang C, Wei X, Chen C, Cao K, Li Y, Jiao Q, Ding J, Zhou J, Fleming BC Chen Q, Shang X, Wei L. Indian hedgehog in synovial fluid is a novel marker for early cartilage lesions in human knee joint. Int J Mol Sci. 2014; 15(5):7250-65.

8. Zhou J, Chen Q, Lanske B, Fleming BC, Terek R, Wei X, Zhang G, Wang S, Li $\mathrm{K}$, Wei L. Disrupting the Indian hedgehog signaling pathway in vivo attenuates surgically induced osteoarthritis progression in Col2a1-CreERT2; Ihhfl/fl mice. Arthritis Res Ther. 2014;16(1):R11.

9. Coventry S, Kapur RP, Siebert JR. Cyclopamine-induced holoprosencephaly and associated craniofacial malformations in the golden hamster: anatomic and molecular events. Pediatr Dev Pathol. 1998;1(1):29-41.

10. Cordero D, Marcucio R, Hu D, Gaffield W, Tapadia M, Helms JA. Temporal perturbations in sonic hedgehog signaling elicit the spectrum of holoprosencephaly phenotypes. J Clin Invest. 2004:114(4):485-94.

11. Lipinski RJ, Hutson PR, Hannam PW, Nydza RJ, Washington IM, Moore RW, Girdaukas GG, Peterson RE, Bushman W. Dose- and route-dependent teratogenicity, toxicity, and pharmacokinetic profiles of the hedgehog signaling antagonist cyclopamine in the mouse. Toxicol Sci. 2008;104(1): 189-97.

12. Lipinski RJ, Dengler E, Kiehn M, Peterson RE, Bushman W. Identification and characterization of several dietary alkaloids as weak inhibitors of hedgehog signaling. Toxicol Sci. 2007;100(2):456-63.

13. Lipinski RJ, Song C, Sulik KK, Everson JL, Gipp JJ, Yan D, Bushman W, Rowland IJ. Cleft lip and palate results from hedgehog signaling antagonism in the mouse: phenotypic characterization and clinical implications. Birth Defects Res A Clin Mol Teratol. 2010;88(4):232-40.

\section{Consent for publication}

Not applicable 
14. Lipinski RJ, Bushman W. Identification of hedgehog signaling inhibitors with relevant human exposure by small molecule screening. Toxicol in Vitro. 2010;24(5):1404-9.

15. Agnusdei D, Bufalino L. Efficacy of ipriflavone in established osteoporosis and long-term safety [J]. Calcif Tissue Int. 1997;61(Suppl 1):S23-7.

16. Gennari C, Adami S, Agnusdei D, Bufalino L, Cervetti R, Crepaldi G, Di Marco C, Di Munno O, Fantasia L, Isaia GC, Mazzuoli GF, Ortolani S, Passeri M, Serni $U$, Vecchiet L. Effect of chronic treatment with Ipriflavone in postmenopausal women with low bone mass. Calcif Tissue Int. 1997; 61(Suppl 1):S19-22.

17. Gennari C, Agnusdei D, Crepaldi G, Isaia G, Mazzuoli G, Ortolani S, Bufalino $L$, Passeri M. Effect of Ipriflavone--a synthetic derivative of natural isoflavones--on bone mass loss in the early years after menopause. Menopause. 1998;5(1):9-15

18. Ohta H, Komukai S, Makita K, Masuzawa T, Nozawa S. Effects of 1-year ipriflavone treatment on lumbar bone mineral density and bone metabolic markers in postmenopausal women with low bone mass. Horm Res. 1999; 51(4):178-83.

19. Alexandersen P, Toussaint A, Christiansen C, Devogelaer JP, Roux C, Fechtenbaum J, Gennari C, Reginster JY. Ipriflavone in the treatment of postmenopausal osteoporosis: a randomized controlled trial. JAMA. 2001; 285(11):1482-8.

20. Hooshmand S, Khalil DA, Murillo G, Singletary K, Kamath SK, Arjmandi BH. The combination of genistin and ipriflavone prevents mammary tumorigenesis and modulates lipid profile. Clin Nutr. 2008;27(4):643-8.

21. Lee DY, Chung HJ, Choi YH, Lee U, Kim SH, Lee I, Lee MG. Pharmacokinetics of ipriflavone and its two metabolites, M1 and M5, after the intravenous and oral administration of ipriflavone to rat model of diabetes mellitus induced by streptozotocin. Eur J Pharm Sci. 2009;38(5):465-71.

22. Kim SH, Lee MG. Pharmacokinetics of ipriflavone, an isoflavone derivative, after intravenous and oral administration to rats hepatic and intestinal firstpass effects. Life Sci. 2002;70(11):1299-315.

23. Ma XH, Xu Y, Liu TP. High performance liquid chromatographic method for the determination of ipriflavone in serum and its pharmacokinetic study in healthy volunteers. Yao Hsueh Hsueh Pao - Acta Pharmaceutica Sinica. 1997;32(6):470-2.

24. Wei L, Sun X, Kanbe K, Wang Z, Sun C, Terek R, Chen Q. Chondrocyte death induced by pathological concentration of chemokine stromal cell-derived factor-1. J Rheumatol. 2006;33(9):1818-26.

25. Wei L, Sun XJ, Terek R, Chen Q. CD95 induced osteoarthritic chondrocytes apoptosis and necrosis: dependency on p38 mitogen-activated protein kinase. Arthritis Res Ther. 2006:8(2):R37.

26. Jay GD, Fleming BC, Watkins BA, McHugh KA, Anderson SC, Zhang LX, Teeple E, Waller KA, Elsaid KA. Prevention of cartilage degeneration and restoration of chondroprotection by lubricin tribosupplementation in the rat following anterior cruciate ligament transection. Arthritis Rheum. 2010;62:2382-91.

27. Glasson SS, Chambers MG, Van Den Berg WB, Little CB. The OARSI histopathology initiative - recommendations for histological assessments of osteoarthritis in the mouse. Osteoarthr Cartil. 2010;18(Suppl 3):S17-23.

28. Young B, Minugh-Purvis N, Shimo T, St-Jacques B, Iwamoto M, EnomotoIwamoto M, Koyama E, Pacifici M. Indian and sonic hedgehogs regulate synchondrosis growth plate and cranial base development and function. Dev Biol. 2006:299(1):272-82.

29. Vortkamp A, Lee K, Lanske B, Segre GV, Kronenberg HM, Tabin CJ. Regulation of rate of cartilage differentiation by Indian hedgehog and PTHrelated protein.[see comment]. Science. 1996;273(5275):613-22.

30. Kobayashi T, Soegiarto DW, Yang Y, Lanske B, Schipani E, McMahon AP, Kronenberg HM. Indian hedgehog stimulates periarticular chondrocyte differentiation to regulate growth plate length independently of PTHrP. J Clin Invest. 2005;115(7):1734-42.

31. Rutges JPHJ, Duit RA, Kummer JA, Oner FC, van Rijen $M H$, Verbout AJ, Castelein RM, Dhert WJA, Creemers LB. Hypertrophic differentiation and calcification during intervertebral disc degeneration. Osteoarthr Cartil. 2010; 18(11):1487-95.

32. Kimura T. Progress of research in osteoarthritis. An overview of the recent knowledge on osteoarthritis: pathogenesis, evaluation and therapies. Clin Calcium. 2009;19(11):1565-71.

33. Abidi A. Hedgehog signaling pathway: a novel target for cancer therapy: vismodegib, a promising therapeutic option in treatment of basal cell carcinomas. Indian J Pharmacol. 2014:46(1):3-12.
34. Kimura H, Ng JM, Curran T. Transient inhibition of the hedgehog pathway in young mice causes permanent defects in bone structure. Cancer Cell. 2008;13(3):249-60.

35. Feldmann G, Fendrich V, McGovern K, Bedja D, Bisht S, Alvarez H, Koorstra JB, Habbe N, Karikari C, Mullendore M, Gabrielson KL, Sharma R, Matsui W, Maitra A. An orally bioavailable small-molecule inhibitor of hedgehog signaling inhibits tumor initiation and metastasis in pancreatic cancer. Mol Cancer Ther. 2008;7(9):2725-35.

36. Bowles DW, Keysar SB, Eagles JR, Wang G, Glogowska MJ, McDermott JD, Le PN, Gao D, Ray CE, Rochon PJ, Roop DR, Tan AC, Serracino HS, Jimeno A. A pilot study of cetuximab and the hedgehog inhibitor IPI-926 in recurrent/ metastatic head and neck squamous cell carcinoma. Oral Oncol. 2016;53:74-9.

37. Yang J, Andre $P, Y e L$, Yang $Y Z$. The hedgehog signalling pathway in bone formation. Int J Oral Sci. 2015;7(2):73-9.

38. Park HL, Bai C, Platt KA, Matise MP, Beeghly A, Hui CC, Nakashima M, Joyner AL. Mouse Gli1 mutants are viable but have defects in $\mathrm{SHH}$ signaling in combination with a Gli2 mutation [J]. Development. 2000;127(8):1593-605.

39. Kesper DA, Didt-Koziel L, Vortkamp A. Gli2 activator function in preosteoblasts is sufficient to mediate Ihh-dependent osteoblast differentiation, whereas the repressor function of $\mathrm{Gli} 2$ is dispensable for endochondral ossification [J]. Dev Dyn. 2010;239(6):1818-26.

40. Shimoyama A, Wada M, Ikeda F, Hata K, Matsubara T, Nifuji A, Noda M, Amano K, Yamaguchi A, Nishimura R, Yoneda T. Ihh/Gli2 signaling promotes osteoblast differentiation by regulating Runx2 expression and function. Mol Biol Cell. 2007;18(7):2411-8

41. Wei L, Fleming BC, Sun X, Teeple E, Wu W, Jay GD, Elsaid KA, Luo J, Machan JT, Chen Q. Comparison of differential biomarkers of osteoarthritis with and without posttraumatic injury in the Hartley guinea pig model. J Orthop Res. 2010;28:900-6.

\section{Ready to submit your research? Choose BMC and benefit from:}

- fast, convenient online submission

- thorough peer review by experienced researchers in your field

- rapid publication on acceptance

- support for research data, including large and complex data types

- gold Open Access which fosters wider collaboration and increased citations

- maximum visibility for your research: over $100 \mathrm{M}$ website views per year

At BMC, research is always in progress.

Learn more biomedcentral.com/submissions 